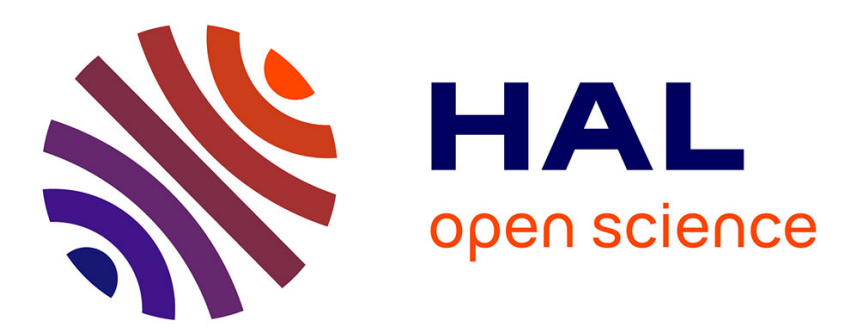

\title{
Output-Feedback H2/Hinf Control of a Class of Networked Fault Tolerant Control Systems
}

Samir Aberkane, Jean-Christophe Ponsart, Dominique Sauter

\section{To cite this version:}

Samir Aberkane, Jean-Christophe Ponsart, Dominique Sauter. Output-Feedback H2/Hinf Control of a Class of Networked Fault Tolerant Control Systems. Asian Journal of Control, 2008, 10 (1), pp.24-33. 10.1002/asjc.4 . hal-00201093

\section{HAL Id: hal-00201093 \\ https://hal.science/hal-00201093}

Submitted on 23 Dec 2007

HAL is a multi-disciplinary open access archive for the deposit and dissemination of scientific research documents, whether they are published or not. The documents may come from teaching and research institutions in France or abroad, or from public or private research centers.
L'archive ouverte pluridisciplinaire HAL, est destinée au dépôt et à la diffusion de documents scientifiques de niveau recherche, publiés ou non, émanant des établissements d'enseignement et de recherche français ou étrangers, des laboratoires publics ou privés. 


\title{
Output-Feedback $\mathcal{H}_{2} / \mathcal{H}_{\infty}$ Control of a Class of Networked Fault Tolerant Control Systems
}

\author{
S. Aberkane*, J. C. Ponsart and D. Sauter \\ CRAN - CNRS UMR 7039 \\ Université Henri Poincaré, Nancy 1, BP 239, \\ F-54506 Vandœuvre-lès-Nancy Cedex \\ Tel.: +33 3836844 80, Fax: +33383684462 \\ * e-mail: samir.aberkane@cran.uhp-nancy.fr
}

September 11, 2007

This paper deals with static output feedback control of a class of discrete-time Networked Control Systems (NCSs) subject to random failures and random delays. The different random processes are modeled as Markovian chains, and the resulting closed-loop system belongs to the class of discretetime Markovian Jump Linear Systems (MJLS). The main contribution is to formulate conditions for multi-performance design related to this class of stochastic hybrid systems. The specifications and objectives under consideration include stochastic stability, $\mathcal{H}_{2}$ and $\mathcal{H}_{\infty}$ performances. Results are formulated as matrix inequalities. A numerical algorithm is provided and its running is illustrated on a numerical example.

Keywords: Fault Tolerant Control - Networked Systems - Markovian Jumping Parameters - Output Feedback.

\section{Introduction}

Networked control systems (NCSs) are feedback control loops closed through a real time network. That is, in NCSs, communication networks are used to exchange informations and control signals (reference input, plant output, control input,...etc.) between control system components (sensors, controllers, actuators,...etc). The main advantages of NCSs are low cost, reduced weight, simple installation and maintenance, and high reliability. As a result, NCSs have great potential in application in complex advanced technological systems such as vehicles, aircrafts, spacecrafts ...etc [30]. At the same time, these complex systems could have various consequences in the event of component failures. Therefore, it is very important to consider the safety and fault tolerance of such systems at the design stage. For these safety-critical systems, Fault Tolerant Control Systems (FTCS) have been developed to meet these essential objectives. FTCS have been a subject of great practical importance, which has attracted a lot of interest for the last three decades. A bibliographical review on reconfigurable fault tolerant control systems can be found in [37].

Despite the advantages and potentials, communication networks in control loops make the analysis and design of NCSs complicated. One main issue is the network induced delays, which occur when sensors, actuators, and controllers exchange data across the network. The delays may be constant, time-varying, and in most cases, random. It is known that the occurrence of delay degrades the stability and control performances of closed-loop control systems. In [22], the stability analysis and control design of NCSs were studied when the network-induced delay at each sampling instant is random and less than one sampling time. In [36], the stability of NCSs was analyzed by a hybrid system approach when the induced delay is deterministic (constant or time-varying) and the controller gain is constant; and in [20], a switched system approach was used to study the stability of NCSs. In 
[33], the maximum of the network-induced delay preserving the closed-loop stability for a given plant and controller was considered. In [32], the network-induced delay is assumed to be time-varying and less than one sampling time. It is noticed that in all the aforementioned papers, the plant is in the continuous-time domain. For the discrete-time case, in [19] and [31], the network-induced random delays were modeled as Markov chains such that the closed-loop system is a jump linear system with one mode. The class of Markovian jump linear systems (MJLS) has attracted increasing attention in the recent literature. Markovian jump systems are those having transition between models determined by a Markov chain. It is very appropriate to model plants whose structures are subject to random abrupt changes due to component failures or repairs, sudden environmental changes, abrupt variations of the operating point of a nonlinear plant, changing subsystem interconnections, and so on. The theory of stability, optimal control and $\mathcal{H}_{2} / \mathcal{H}_{\infty}$ control, as well as important applications of such systems, can be found in several papers in the current literature, for instance in $[4,5,6,8,10,11,12,13,15,16]$ for continuous-time case, and [9] for the discrete-time case. Fault tolerant control issues were also considered in the same framework, for instance in [1, 2, 3, 21, 25, 26, 28].

On the other hand, one of the most challenging open problems in control theory is the synthesis of fixed-order or static output feedback controllers that meet desired performances and specifications [29]. Among all variations of this problem, this note is concerned with the problem of static output feedback control of discrete-time NCSs. This problem is addressed under a MJLS framework. Most of the developed results in the field of MJLS control are obtained under the restrictive assumption of control with complete state observation (Markov and system states). However, the access to the system mode may not be possible in some circumstances, which limits the use of such controllers. For instance, if the changes in the Markov chain are associated to failures of components of non-critical significance, or more generally if some changes are difficult to measure, it is quite possible that the associated Markov states are not accessible to controller. To design mode-independent controllers, two approaches can be adopted. The first one employs a constant Lyapunov function, which makes the design problem formulated as a linear matrix inequality (LMI) problem. The second one uses mode-dependent Lyapunov function and therefore leads to less conservative results. To the best of our knowledge, to date the problem of mode-independent output-feedback $\mathcal{H}_{2} / \mathcal{H}_{\infty}$ control of discretetime NCSs has not yet been fully addressed. Our approach belongs to the class of methods that use mode-dependent Lyapunov functions.

This paper is organized as follows: Section 2 describes the dynamical model of the system with appropriately defined random processes. A brief summary of basic stochastic terms, results and definitions are given in Section 3. Section 4 addresses the stochastic stabilization of NCSs. Sections 5 considers the $\mathcal{H}_{2} / \mathcal{H}_{\infty}$ control problem for the output feedback. In Section 6, a numerical algorithm based on nonconvex optimization is provided and its running is illustrated on a classical example from literature. Finally, a conclusion is given in Section 7.

Notations. The notations in this paper are quite standard. $\mathbb{R}^{m \times n}$ is the set of $m$-by- $n$ real matrices. $A^{\prime}$ is the transpose of the matrix $A$. The notation $X \geq Y(X>Y$, respectively), where $X$ and $Y$ are symmetric matrices, means that $X-Y$ is positive semi-definite (positive definite, respectively); $\mathbb{I}$ and $\mathbf{0}$ are identity and zero matrices of appropriate dimensions, respectively; $\mathcal{E}\{\cdot\}$ denotes the expectation operator with respect to some probability measure $P ; L^{2}[0, \infty)$ stands for the space of squaresummable vector functions over the interval $[0, \infty) ;\|\cdot\|$ refers to either the Euclidean vector norm or the matrix norm, which is the operator norm induced by the standard vector norm; $\|\cdot\|_{2}$ stands for the norm in $L^{2}[0, \infty)$; while $\|\cdot\|_{\mathcal{E}_{2}}$ denotes the norm in $L^{2}((\Omega, \mathcal{F}, P),[0, \infty)) ;(\Omega, \mathcal{F}, P)$ is a probability space. In block matrices, $\star$ indicates symmetric terms: $\left[\begin{array}{cc}A & B \\ B^{\prime} & C\end{array}\right]=\left[\begin{array}{cc}A & \star \\ B^{\prime} & C\end{array}\right]=\left[\begin{array}{cc}A & B \\ \star & C\end{array}\right]$. 


\section{System Modeling}

Consider the following class of dynamical systems in a given fixed complete probability space $(\Omega, \mathcal{F}, P)$ :

$$
\varphi:\left\{\begin{array}{l}
x_{k+1}=A_{x}\left(\eta_{k}\right) x_{k}+B_{u}\left(\eta_{k}\right) u\left(y_{k}, k\right)+B_{w}\left(\eta_{k}\right) w_{k} \\
y_{k}=C_{y} x_{k} \\
z_{k}=C_{z}\left(\eta_{k}\right) x_{k}+D_{z}\left(\eta_{k}\right) u\left(y_{k}, k\right)
\end{array}\right.
$$

where $x_{k} \in \mathbb{R}^{n}$ is the system state, $u\left(y_{k}, k\right) \in \mathbb{R}^{r}$ is the system input, $y_{k} \in \mathbb{R}^{q}$ is the system measured output, $w_{k} \in \mathbb{R}^{m}$ is the system external disturbance which belongs to $L^{2}[0, \infty), z_{k}$ is the controlled output which belongs to $L^{2}((\Omega, \mathcal{F}, P),[0, \infty))$ and $\left\{\eta_{k}, k \geq 0\right\}$ denotes the state of the random process describing the failures. It is assumed that $\eta_{k}$ is a measurable discrete-time Markov process taking values on a finite set $\beth=\{1, \ldots, \nu\}$. For the failure process $\eta_{k}$, the known one-step transition probability from state $i$ to state $l(i, l \in \beth)$ is given by $\alpha_{i l}$, i.e.

$$
\alpha_{i l}=P\left\{\eta_{k+1}=l \mid \eta_{k}=i\right\}
$$

It is also assumed that there are random but bounded delays from the sensor to the controller

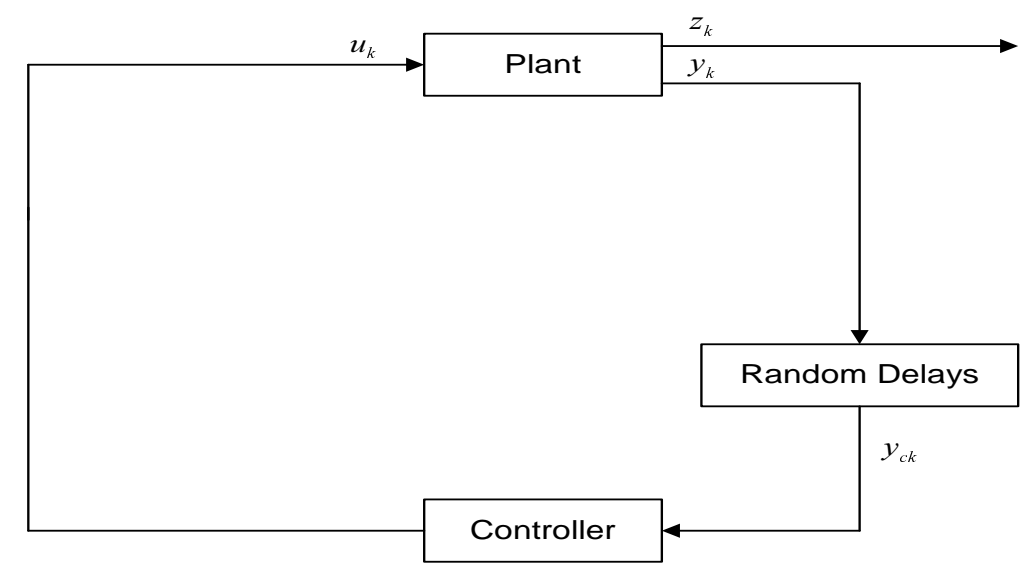

Figure 1: Control over networks

(Figure-1). The mode-independent static output feedback control law is

$$
\varphi_{s}:\left\{u\left(y_{k}, r_{s k}, k\right)=\mathcal{K} y_{c k}=\mathcal{K} C_{y} x_{k-r_{s k}}\right.
$$

where $\left\{r_{s k}\right\}$ is a bounded random integer sequence with $0 \leq r_{s k} \leq d_{s}<\infty$, and $d_{s}$ is the finite delay bound.

If we augment the state variable

$$
\tilde{x}_{k}=\left[\begin{array}{llll}
x_{k}^{\prime} & x_{k-1}^{\prime} & \ldots & x_{k-d_{s}}^{\prime}
\end{array}\right]^{\prime}
$$

where $\tilde{x}_{k} \in \mathbb{R}^{\left(d_{s}+1\right) n}$, then the closed-loop system is given by

$$
\varphi_{c l}:\left\{\begin{array}{l}
\tilde{x}_{k+1}=\left(\tilde{A}_{x}\left(\eta_{k}\right)+\tilde{B}_{u}\left(\eta_{k}\right) \mathcal{K} \tilde{C}_{y}\left(r_{s k}\right)\right) \tilde{x}_{k}+\tilde{B}_{w}\left(\eta_{k}\right) w_{k} \\
y_{k}=\tilde{C}_{y}\left(r_{s k}\right) \tilde{x}_{k} \\
z_{k}=\left(\tilde{C}_{z}\left(\eta_{k}\right)+D_{z}\left(\eta_{k}\right) \mathcal{K} \tilde{C}_{y}\left(r_{s k}\right)\right) \tilde{x}_{k}
\end{array}\right.
$$

where

$$
\tilde{A}_{x}\left(\eta_{k}\right)=\left[\begin{array}{ccccc}
A_{x}\left(\eta_{k}\right) & \mathbf{0} & \ldots & \mathbf{0} & \mathbf{0} \\
\mathbb{I} & \mathbf{0} & \ldots & \mathbf{0} & \mathbf{0} \\
\mathbf{0} & \mathbb{I} & \ldots & \mathbf{0} & \mathbf{0} \\
\vdots & \vdots & \ddots & \vdots & \vdots \\
\mathbf{0} & \mathbf{0} & \ldots & \mathbb{I} & \mathbf{0}
\end{array}\right], \tilde{B}_{u}\left(\eta_{k}\right)=\left[\begin{array}{c}
B_{u}\left(\eta_{k}\right) \\
\mathbf{0} \\
\mathbf{0} \\
\vdots \\
\mathbf{0}
\end{array}\right], \tilde{B}_{w}\left(\eta_{k}\right)=\left[\begin{array}{c}
B_{w}\left(\eta_{k}\right) \\
\mathbf{0} \\
\mathbf{0} \\
\vdots \\
\mathbf{0}
\end{array}\right]
$$




$$
\begin{gathered}
\tilde{C}_{y}\left(r_{s k}\right)=\left[\begin{array}{lllllll}
\mathbf{0} & \ldots & \mathbf{0} & C_{y} & \mathbf{0} & \ldots & \mathbf{0}
\end{array}\right] \\
\tilde{C}_{z}\left(\eta_{k}\right)=\left[\begin{array}{lllllll}
C_{z}\left(\eta_{k}\right) & \ldots & \mathbf{0} & \mathbf{0} & \mathbf{0} & \ldots & \mathbf{0}
\end{array}\right]
\end{gathered}
$$

and $\tilde{C}_{y}\left(r_{s k}\right)$ has all elements being zero except for the $\left(r_{s k}+1\right)-$ th block being the matrice $C_{y}$. One of the difficulties with this approach is how to model the $r_{s k}$ sequence. One way is to model the transitions of the random delays $r_{s k}$ as a finite state Markov process where $r_{s k} \in S=\left\{0, \ldots, d_{s}\right\}$ $[19,31,35]$. In this case we have

$$
P\left\{r_{s(k+1)}=j \mid r_{s k}=i\right\}=p_{i j}
$$

where $0 \leq i, j \leq d_{s}$. This model is quite general, communication package loss in the network can be included naturally as explained below [31]. The assumption here is that the controller will always use the most recent data. Thus, if we have $y_{\left(k-r_{s k}\right)}$ at step $k$, but there is no new information coming at step $k+1$ (data could be lost or there is a longer delay), then we at least have $y_{\left(k-r_{s k}\right)}$ available for feedback. So, in our model of the system in Figure 1, the delay $r_{s k}$ can increase at most by 1 each step, and we constrain

$$
P\left\{r_{s(k+1)}>r_{s k}+1\right\}=0
$$

However, the delay $r_{s k}$ can decrease as many steps as possible. Decrement of $r_{s k}$ models communication package loss in the network, or disregarding old data if we have newer data coming at the same time. Hence the structured transition probability matrix is

$$
P_{s}=\left[\begin{array}{cccccc}
p_{00} & p_{01} & 0 & 0 & \ldots & 0 \\
p_{10} & p_{11} & p_{12} & 0 & \ldots & 0 \\
\vdots & \vdots & \vdots & \vdots & \ddots & \vdots \\
\vdots & \vdots & \vdots & \vdots & \vdots & p_{\left(d_{s}-1\right) d_{s}} \\
p_{d_{s} 0} & p_{d_{s} 1} & p_{d_{s} 2} & p_{d_{s} 3} & \ldots & p_{d_{s} d_{s}}
\end{array}\right]
$$

where

$$
0 \leq p_{i j} \leq 1 \text { and } \sum_{j=0}^{d_{s}} p_{i j}=1
$$

because each row represents the transition probabilities from a fixed state to all the states. The diagonal elements are the probabilities of data coming in sequence with equal delays. The elements above the diagonal are the probabilities of encountering longer delays, and the elements below the diagonal indicate package loss or disregarding old data.

\section{Definitions and Basic Results}

In this section, we will first give some basic definitions related to stochastic stability notions and then we will summarize some results about stochastic stabilizability of the discrete-time NCS subject to random failures and delays.

We introduce the following stability definition for discrete-time jump linear systems.

Definition 1. The system (4) with $u_{k} \equiv 0, w_{k} \equiv 0$, is said to be stochastically stable (SS), if for every initial state $\left(\tilde{x}_{0}, r_{s 0}, \eta_{0}\right)$, the following holds:

$$
\mathcal{E}\left\{\sum_{k=0}^{\infty}\left\|\tilde{x}_{k}\left(\tilde{x}_{0}, r_{s 0}, \eta_{0}\right)\right\|^{2} \mid \tilde{x}_{0}, r_{s 0}, \eta_{0}\right\}<\infty
$$

The following proposition gives a necessary and sufficient condition for the (SS) of system (4).

Proposition 1. The following statements are equivalent:

i) System (4) is stochastically stabilizable by $\varphi_{s}$; 
ii) The matrix inequalities

$$
\bar{A}_{i j}^{\prime} \overline{\mathcal{P}}_{i j} \bar{A}_{i j}-\mathcal{P}_{i j}=\beth_{i j}<0, \quad \forall i \in \beth, j \in S .
$$

are feasible for some matrices $\mathcal{K}$ and $\mathcal{P}_{i j}>0$, where

$$
\bar{A}_{i j}=\tilde{A}_{x i}+\tilde{B}_{u i} \mathcal{K} \tilde{C}_{y j} ; \quad \overline{\mathcal{P}}_{i j}=\sum_{v=1}^{d_{s}} p_{j v} \sum_{m=1}^{\nu} \alpha_{i m} \mathcal{P}_{m v}
$$

iii) For any given $\mathbf{Q}=\left(\mathcal{Q}_{11}, \ldots, \mathcal{Q}_{i j}, \ldots, \mathcal{Q}_{\nu d_{s}}\right)$ with $\mathcal{Q}_{i j}>0$, there exist a unique

$\mathbf{P}=\left(\mathcal{P}_{11}, \ldots, \mathcal{P}_{i j}, \ldots, \mathcal{P}_{\nu d_{s}}\right)$ with $\mathcal{P}_{i j}>0$ satisfying the following coupled Lyapunov equations

$$
\bar{A}_{i j}^{\prime} \overline{\mathcal{P}}_{i j} \bar{A}_{i j}-\mathcal{P}_{i j}+\mathcal{Q}_{i j}=0 \quad \forall i \in \beth, j \in S .
$$

Proof. The proof of this proposition follows the same lines as for the proof of stability results in [9, 34], except here we consider two Markovian processes, while in the aforementioned references, the authors consider a single Markov process.

\subsection{Matrix Ellipsoids}

Through this note, a particular set of matrices is used. Due to the notations and by extension of the notion of $\mathbb{R}^{n}$ ellipsoids, these sets are referred to as matrix ellipsoids of $\mathbb{R}^{(m \times p)}$.

Definition 2. [23, 24] Given three matrices $\mathbb{X} \in \mathbb{S}^{q}, \mathbb{Y} \in \mathbb{R}^{q \times r}$ and $\mathbb{Z} \in \mathbb{S}^{r}$, the $\{\mathbb{X}, \mathbb{Y}, \mathbb{Z}\}$-ellipsoid of $\mathbb{R}^{r \times q}$ is the set of matrices $\mathcal{K}$ satisfying the following matrix inequalities:

$$
\mathbb{Z}>0 ; \quad\left[\begin{array}{ll}
\mathbb{I} & \mathcal{K}^{\prime}
\end{array}\right]\left[\begin{array}{cc}
\mathbb{X} & \mathbb{Y} \\
\star & \mathbb{Z}
\end{array}\right]\left[\begin{array}{c}
\mathbb{I} \\
\mathcal{K}
\end{array}\right] \leq 0
$$

By definition, $\mathcal{K}_{0}=-\mathbb{Z}^{-1} \mathbb{Y}^{\prime}$ is the center of the ellipsoid and $R=\mathcal{K}_{0}^{\prime} \mathbb{Z} \mathcal{K}_{0}-\mathbb{X}$ is the radius. Inequality (11) can also be written as

$$
\mathbb{Z}>0 ; \quad\left(\mathcal{K}-\mathcal{K}_{0}\right)^{\prime} \mathbb{Z}\left(\mathcal{K}-\mathcal{K}_{0}\right) \leq R
$$

This definition shows that matrix ellipsoids are special cases of matrix sets defined by quadratic matrix inequality. Some properties of these sets are:

i) A matrix ellipsoid is a convex set;

ii) the $\{\mathbb{X}, \mathbb{Y}, \mathbb{Z}\}$-ellipsoid is nonempty iff the radius $(R \geq 0)$ is positive semi definite. This property can also be expressed as

$$
\mathbb{X} \leq \mathbb{Y} \mathbb{Z}^{-1} \mathbb{Y}^{\prime}
$$

\section{Stochastic Stabilization}

In this section, we shall address the problem of finding all static compensators $\left(\varphi_{s}\right)$, as defined in section 2 , such that the closed loop system $\left(\varphi_{c l}\right)$ becomes stochastically stable. To this end, we use proposition 1 and the idea of synthesizing convex sets of controllers [23, 24] to get the following necessary and sufficient conditions for the (SS) of the system (4).

Proposition 2. System (4) is stochastically stabilised by static output-feedback compensator $\left(\varphi_{s}\right)$ if and only if there exists matrices $\mathcal{P}_{i j}=\mathcal{P}_{i j}^{\prime}>0, \mathbb{X} \in \mathbb{S}^{q}, \mathbb{Y} \in \mathbb{R}^{q \times r}$ and $\mathbb{Z}=\mathbb{Z}^{\prime}>0$ that simultaneously satisfy the following LMI constraints

$$
\left[\begin{array}{cc}
\mathbb{I} & \mathbf{0} \\
\tilde{A}_{x i} & \tilde{B}_{u i}
\end{array}\right]^{\prime}\left[\begin{array}{cc}
-\mathcal{P}_{i j} & \mathbf{0} \\
\star & \overline{\mathcal{P}}_{i j}
\end{array}\right]\left[\begin{array}{cc}
\mathbb{I} & \mathbf{0} \\
\tilde{A}_{x i} & \tilde{B}_{u i}
\end{array}\right]<\left[\begin{array}{cc}
\tilde{C}_{y j} & \mathbf{0} \\
\mathbf{0} & \mathbb{I}
\end{array}\right]^{\prime}\left[\begin{array}{cc}
\mathbb{X} & \mathbb{Y} \\
\star & \mathbb{Z}
\end{array}\right]\left[\begin{array}{cc}
\tilde{C}_{y j} & \mathbf{0} \\
\mathbf{0} & \mathbb{I}
\end{array}\right]
$$


and the nonlinear inequality constraint

$$
\mathbb{X} \leq \mathbb{Y} \mathbb{Z}^{-1} \mathbb{Y}^{\prime}
$$

$\forall i \in \beth, j \in S$.

Let $\left\{\mathcal{P}_{i j}, \mathbb{X}, \mathbb{Y}, \mathbb{Z}\right\}$ be a solution, then the nonempty $\{\mathbb{X}, \mathbb{Y}, \mathbb{Z}\}$-ellipsoid is a set of stabilizing gains.

\section{Proof.}

Sufficiency. Assume that the constraints (14)-(15) are satisfied for some $\left\{\mathcal{P}_{i j}, \mathbb{X}, \mathbb{Y}, \mathbb{Z}\right\}$ matrices. Due to the properties of matrix ellipsoids, the $\{\mathbb{X}, \mathbb{Y}, \mathbb{Z}\}$-ellipsoid is nonempty. Take any element $\mathcal{K}$. The LMI (14) implies that for all $\left(\begin{array}{cc}\tilde{x}_{k}^{\prime} & u_{k}^{\prime}\end{array}\right) \neq 0$

$$
\left(\begin{array}{c}
\tilde{x}_{k} \\
\tilde{A}_{x i} \tilde{x}_{k}+\tilde{B}_{u i} u_{k}
\end{array}\right)^{\prime}\left[\begin{array}{cc}
-\mathcal{P}_{i j} & \mathbf{0} \\
\star & \overline{\mathcal{P}}_{i j}
\end{array}\right]\left(\begin{array}{c}
\tilde{x}_{k} \\
\tilde{A}_{x i} \tilde{x}_{k}+\tilde{B}_{u i} u_{k}
\end{array}\right)<\left(\begin{array}{c}
\tilde{C}_{y j} \tilde{x}_{k} \\
u_{k}
\end{array}\right)^{\prime}\left[\begin{array}{cc}
\mathbb{X} & \mathbb{Y} \\
\star & \mathbb{Z}
\end{array}\right]\left(\begin{array}{c}
\tilde{C}_{y j} \tilde{x}_{k} \\
u_{k}
\end{array}\right)
$$

Definition 2 implies that for all nonzero trajectories

$$
\tilde{x}_{k}^{\prime} \rrbracket_{i j} \tilde{x}_{k}<y_{k}^{\prime}\left[\mathbb{I} \mathcal{K}^{\prime}\right]\left[\begin{array}{cc}
\mathbb{X} & \mathbb{Y} \\
\star & \mathbb{Z}
\end{array}\right]\left[\begin{array}{c}
\mathbb{I} \\
\mathcal{K}
\end{array}\right] y_{k} \leq 0
$$

$\forall i \in \beth$ and $j \in S$.

Then, the closed-loop stochastic stability is assessed by proposition 1 for the quadratic stochastic Lyapunov function $\vartheta\left(\eta_{k}, r_{s k}\right)=\tilde{x}_{k}^{\prime} \mathcal{P}\left(\eta_{k}, r_{s k}\right) \tilde{x}_{k}$.

Necessity. Assume $\mathcal{K}$ is a stabilizing static output feedback gain and $\vartheta\left(\eta_{k}, r_{s k}\right)=\tilde{x}_{k}^{\prime} \mathcal{P}\left(\eta_{k}, r_{s k}\right) \tilde{x}_{k}$ is a stochastic Lyapunov function. Then from proposition 1 , we have

$$
\left[\mathcal{K} \tilde{C}_{y j} \quad-\mathbb{I}\right]\left(\begin{array}{l}
\tilde{x}_{k} \\
u_{k}
\end{array}\right)=0 \Rightarrow\left(\begin{array}{c}
\tilde{x}_{k} \\
u_{k}
\end{array}\right)^{\prime}\left[\begin{array}{cc}
\mathbb{I} & \mathbf{0} \\
\tilde{A}_{x i} & \tilde{B}_{u i}
\end{array}\right]^{\prime}\left[\begin{array}{cc}
-\mathcal{P}_{i j} & \mathbf{0} \\
\star & \overline{\mathcal{P}}_{i j}
\end{array}\right]\left[\begin{array}{cc}
\mathbb{I} & \mathbf{0} \\
\tilde{A}_{x i} & \tilde{B}_{u i}
\end{array}\right]\left(\begin{array}{l}
\tilde{x}_{k} \\
u_{k}
\end{array}\right)<0
$$

$\forall i \in \beth$ and $j \in S$.

Applying the well known Finsler Lemma [27], there exist scalars $\tau_{i j}$ such that

$\left[\begin{array}{cc}\mathbb{I} & \mathbf{0} \\ \tilde{A}_{x i} & \tilde{B}_{u i}\end{array}\right]^{\prime}\left[\begin{array}{cc}-\mathcal{P}_{i j} & \mathbf{0} \\ \star & \overline{\mathcal{P}}_{i j}\end{array}\right]\left[\begin{array}{cc}\mathbb{I} & 0 \\ \tilde{A}_{x i} & \tilde{B}_{u i}\end{array}\right]<\tau_{i j}\left[\begin{array}{cc}\mathcal{K} \tilde{C}_{y j} & -\mathbb{I}\end{array}\right]^{\prime}\left[\begin{array}{cc}\mathcal{K} \tilde{C}_{y j} & -\mathbb{I}\end{array}\right] \leq \varepsilon\left[\begin{array}{cc}\mathcal{K} \tilde{C}_{y j} & -\mathbb{I}\end{array}\right]^{\prime}\left[\begin{array}{cc}\mathcal{K} \tilde{C}_{y j} & -\mathbb{I}\end{array}\right]$

where $\varepsilon=\max _{i, j}\left(\tau_{i j}\right)$. The inequality (15) is obtained with

$$
\mathbb{X}=\varepsilon \mathcal{K}^{\prime} \mathcal{K}, \quad \mathbb{Y}=-\varepsilon \mathcal{K}^{\prime}, \quad \mathbb{Z}=\varepsilon \mathbb{I}
$$

From (19) we have $\mathbf{0}<\mathbb{Z}$. Hence the proof is complete.

\section{The Control Problem}

\section{$5.1 \quad \mathcal{H}_{\infty}$ Control}

Let us consider the system (1) with

$$
z_{k}=z_{\infty k}=C_{\infty}\left(\eta_{k}\right) x_{k}+D_{\infty}\left(\eta_{k}\right) u\left(y_{k}, k\right)
$$

$z_{\infty k}$ stands for the controlled output related to $\mathcal{H}_{\infty}$ performance.

In this section, we deal with the design of controllers that stochastically stabilize the closed-loop system and guarantee the disturbance rejection, with a certain level $\gamma_{\infty}>0$. This problematic is addressed under a nonconvex optimization framework.

In order to put the $\mathcal{H}_{\infty}$ control problem in a stochastic setting, we bring to bear the space $L^{2}((\Omega, \mathcal{F}, P),[0, \infty))$ of $\mathcal{F}$-measurable processes, $z_{\infty k}$, for which

$$
\left\|z_{\infty}\right\|_{\mathcal{E}_{2}}=\mathcal{E}\left\{\sum_{k=0}^{\infty} z_{\infty k}^{\prime} z_{\infty k}\right\}^{1 / 2}<\infty
$$

The stochastic $\mathcal{H}_{\infty}$ control problem can be stated as follows: 
For a given level on the $\mathcal{H}_{\infty}$ norm, $\gamma_{\infty}$, find a stabilizing static output feedback gain $\mathcal{K}$ such that

$$
\mathcal{E}\left\{\sum_{k=0}^{\infty} z_{\infty k}^{\prime} z_{\infty k}\right\}<\gamma_{\infty}^{2} \sum_{k=0}^{\infty} w_{k}^{\prime} w_{k}
$$

i.e.

$$
\left\|z_{\infty}\right\|_{\mathcal{E}_{2}}<\gamma_{\infty}\|w\|_{2}
$$

In this situation, the closed loop system (4) is said to have an $\mathcal{H}_{\infty}$ performance level $\gamma_{\infty}$ over $[0, \infty)$.

Before introducing our result on $\mathcal{H}_{\infty}$ control for this class of stochastic hybrid systems, let us consider the following proposition which is obtained as a special case of the bounded real lemma of discrete time Markovian jump linear systems [34].

Proposition 3. The system (4) is stochastically stable and $\left\|\varphi_{c l}\right\|_{\infty}<\gamma_{\infty}$ if there exist a matrix $\mathcal{K}$ and symmetric matrices $\mathcal{P}_{i j}>0$ satisfying the following coupled matrix inequalities

$$
\left[\begin{array}{cc}
\bar{A}_{i j}^{\prime} \overline{\mathcal{P}}_{i j} \bar{A}_{i j}-\mathcal{P}_{i j}+\bar{C}_{\infty i j}^{\prime} \bar{C}_{\infty i j} & \bar{A}_{i j}^{\prime} \overline{\mathcal{P}}_{i j} \tilde{B}_{w i} \\
\star & -\left(\gamma_{\infty}^{2} \mathbb{I}-\tilde{B}_{w i}^{\prime} \overline{\mathcal{P}}_{i j} \tilde{B}_{w i}\right)
\end{array}\right]<0
$$

where

$$
\bar{C}_{\infty i j}=\tilde{C}_{\infty i}+D_{\infty i} \mathcal{K} \tilde{C}_{y j}
$$

Now, we are in position to give the result on the solvability of the $\mathcal{H}_{\infty}$ static output feedback control problem. Indeed, proposition 4 gives a nonlinear matrix inequalities characterization of static output feedback compensator $\left(\varphi_{s}\right)$ that stochastically stabilize the closed loop system (4) and ensures (20).

Proposition 4. If there exists matrices $\mathcal{P}_{\infty i j}=\mathcal{P}_{\infty i j}^{\prime}>0, \mathbb{X} \in \mathbb{S}^{q}, \mathbb{Y} \in \mathbb{R}^{q \times r}$ and $\mathbb{Z}=\mathbb{Z}^{\prime}>0$ that simultaneously satisfy the following LMI constraints

$$
\mathbb{M}_{1 i}^{\prime}\left[\begin{array}{cc}
-\mathcal{P}_{\infty i j} & \mathbf{0} \\
\star & \overline{\mathcal{P}}_{\infty i j}
\end{array}\right] \mathbb{M}_{1 i}<\mathbb{M}_{2 i}^{\prime}\left[\begin{array}{cc}
-\mathbb{I} & \mathbf{0} \\
\mathbf{0} & \gamma_{\infty}^{2} \mathbb{I}
\end{array}\right] \mathbb{M}_{2 i}+\mathbb{M}_{3 j}^{\prime}\left[\begin{array}{cc}
\mathbb{X} & \mathbb{Y} \\
\star & \mathbb{Z}
\end{array}\right] \mathbb{M}_{3 j}
$$

and the nonlinear inequality constraint

$$
\mathbb{X} \leq \mathbb{Y} \mathbb{Z}^{-1} \mathbb{Y}^{\prime}
$$

$\forall i \in \beth, j \in S$, where

$$
\mathbb{M}_{1 i}=\left[\begin{array}{ccc}
\mathbb{I} & \mathbf{0} & \mathbf{0} \\
\tilde{A}_{x i} & \tilde{B}_{w i} & \tilde{B}_{u i}
\end{array}\right], \quad \mathbb{M}_{2 i}=\left[\begin{array}{ccc}
\tilde{C}_{\infty i} & \mathbf{0} & D_{\infty i} \\
\mathbf{0} & \mathbb{I} & \mathbf{0}
\end{array}\right], \quad \mathbb{M}_{3 j}=\left[\begin{array}{ccc}
\tilde{C}_{y j} & \mathbf{0} & \mathbf{0} \\
\mathbf{0} & \mathbf{0} & \mathbb{I}
\end{array}\right]
$$

then the $\{\mathbb{X}, \mathbb{Y}, \mathbb{Z}\}$-ellipsoid is a set of stabilizing gains such that

$$
\left\|z_{\infty}\right\|_{\mathcal{E}_{2}}<\gamma_{\infty}\|w\|_{2}
$$

Proof. The proof of this proposition follows the same arguments as for the proof of proposition 2 .

\section{$5.2 \quad \mathcal{H}_{2}$ Control}

Before introducing the main results of this section, let us consider the following definition which represents a generalization of the $\mathcal{H}_{2}$-norm of discrete-time Markovian jump linear systems [7]. Let us consider the system (1) with

$$
z_{k}=z_{2 k}=C_{2}\left(\eta_{k}\right) x_{k}+D_{2}\left(\eta_{k}\right) u\left(y_{k}, k\right)
$$


$z_{2 k}$ stands for the controlled output related to $\mathcal{H}_{2}$ performance.

Definition 3. We define the $\mathcal{H}_{2}$-norm of the $(S S)$ system $\left(\varphi_{c l}\right)$ as

$$
\left\|\varphi_{c l}\right\|_{2}^{2}=\sum_{d=1}^{m} \sum_{i, j}\left\|z_{2 d, i, j}\right\|_{\mathcal{E}_{2}}^{2}
$$

where $z_{2 d, i, j}$ represents the output sequence $\left(z_{20}, z_{21}, \ldots\right)$ when :

a) the input sequence is given by $w=\left(w_{0}, w_{1}, \ldots\right), w_{0}=e_{d}, w_{k}=0, k>0, e_{d} \in \mathbb{R}^{m}$ is the unitary vector formed by one at the $d$-th position and zero elsewhere;

b) $\eta_{0}=\eta_{1}=i, r_{s 0}=r_{s 1}=j$.

From the definition above and using the same arguments as in [7], we can state the following corollary.

Corollary 2. Assume that $\left(\varphi_{c l}\right)$ is $(S S)$ then

i) $\left\|\varphi_{c l}\right\|_{2}^{2}=\sum_{i, j} \operatorname{tr}\left(\tilde{B}_{w i}^{\prime} \mathcal{P}_{o i j} \tilde{B}_{w i}\right)$, where $\mathcal{P}_{o}=\left\{\mathcal{P}_{o 11}, \ldots, \mathcal{P}_{o \nu d_{s}}\right\}$ denotes the observability Grammian, i.e., $\mathcal{P}_{o i j}$ are the unique positive semi definite solutions of the following equations

$$
\bar{A}_{i j}^{\prime} \overline{\mathcal{P}}_{o i j} \bar{A}_{i j}-\mathcal{P}_{o i j}+\bar{C}_{2 i j}^{\prime} \bar{C}_{2 i j}=0
$$

$\forall i \in \beth$ and $j \in S$.

ii) $\left\|\varphi_{c l}\right\|_{2}^{2}<\sum_{i, j} \operatorname{tr}\left(\tilde{B}_{w i}^{\prime} \mathcal{P}_{2 i j} \tilde{B}_{w i}\right)$, where $\mathcal{P}_{2 i j}$ is a positive definite solution of the following matrix inequality

$$
\bar{A}_{i j}^{\prime} \overline{\mathcal{P}}_{2 i j} \bar{A}_{i j}-\mathcal{P}_{2 i j}+\bar{C}_{2 i j}^{\prime} \bar{C}_{2 i j}<0
$$

$\forall i \in \beth$ and $j \in S$.

iii) If there exists positive definite matrices $\mathcal{P}_{2 i j}$ and a matrix $\mathcal{K}$ such that

$$
\begin{gathered}
\sum_{i, j} \operatorname{tr}\left(\tilde{B}_{w i}^{\prime} \mathcal{P}_{2 i j} \tilde{B}_{w i}\right)<\gamma_{2}^{2} \\
\bar{A}_{i j}^{\prime} \overline{\mathcal{P}}_{2 i j} \bar{A}_{i j}-\mathcal{P}_{2 i j}+\bar{C}_{2 i j}^{\prime} \bar{C}_{2 i j}<0
\end{gathered}
$$

$\forall i \in \beth$ and $j \in S$, then $\mathcal{K}$ is a stabilizing gain such that $\left\|\varphi_{c l}\right\|_{2}<\gamma_{2}$.

Using the same framework as for the stochastic stabilization and the $\mathcal{H}_{\infty}$ control problematic, similar $\mathcal{H}_{2}$ control results are obtained and summarized as follows:

Proposition 5. If there exists matrices $\mathcal{P}_{2 i j}=\mathcal{P}_{2 i j}^{\prime}>0, \mathbb{X} \in \mathbb{S}^{q}, \mathbb{Y} \in \mathbb{R}^{q \times r}$ and $\mathbb{Z}=\mathbb{Z}^{\prime}$ that simultaneously satisfy the constraints

$$
\begin{gathered}
\mathbb{X} \leq \mathbb{Y} \mathbb{Z}^{-1} \mathbb{Y}^{\prime} \\
\sum_{i, j} \operatorname{tr}\left(\tilde{B}_{w i}^{\prime} \mathcal{P}_{2 i j} \tilde{B}_{w i}\right)<\gamma_{2}^{2} \\
\mathbb{N}_{1 i}^{\prime}\left[\begin{array}{cc}
-\mathcal{P}_{2 i j} & \mathbf{0} \\
\star & \overline{\mathcal{P}}_{2 i j}
\end{array}\right] \mathbb{N}_{1 i}<-\mathbb{N}_{2 i}^{\prime} \mathbb{N}_{2 i}+\mathbb{N}_{3 j}^{\prime}\left[\begin{array}{cc}
\mathbb{X} & \mathbb{Y} \\
\star & \mathbb{Z}
\end{array}\right] \mathbb{N}_{3 j}
\end{gathered}
$$

$\forall i \in \Xi, j \in S$, where

$$
\mathbb{N}_{1 i}=\left[\begin{array}{cc}
\mathbb{I} & \mathbf{0} \\
\tilde{A}_{x i} & \tilde{B}_{u i}
\end{array}\right], \quad \mathbb{N}_{2 i}=\left[\begin{array}{cc}
\tilde{C}_{2 i} & D_{2 i}
\end{array}\right], \quad \mathbb{N}_{3 j}=\left[\begin{array}{cc}
\tilde{C}_{y j} & \mathbf{0} \\
\mathbf{0} & \mathbb{I}
\end{array}\right]
$$

then the $\{\mathbb{X}, \mathbb{Y}, \mathbb{Z}\}$-ellipsoid is a set of stabilizing gains such that $\left\|\varphi_{c l}\right\|_{2}<\gamma_{2}$.

Proof. The proof of this proposition follows the same arguments as for the proof of proposition 2 . 


\subsection{Multi-Objective Synthesis}

The multi-objective synthesis problem consists in finding a common controller that stochastically stabilizes the system and ensures the different performance levels. This can be stated as follows:

For two given levels on the $\mathcal{H}_{\infty}$ and $\mathcal{H}_{2}$ norms, $\gamma_{\infty}$ and $\gamma_{2}$ respectively, find a stabilizing static output feedback gain $\mathcal{K}$ such that

$$
\begin{gathered}
\left\|z_{\infty}\right\|_{\mathcal{E}_{2}}<\gamma_{\infty}\|w\|_{2} \\
\left\|\varphi_{c l}\right\|_{2}<\gamma_{2}
\end{gathered}
$$

The result is straightforward. It amounts to verify all related matrix inequality constraints.

Corollary 2. If there exists matrices $\mathcal{P}_{\infty i j}, \mathcal{P}_{2 i j}, \mathbb{X} \in \mathbb{S}^{q}, \mathbb{Y} \in \mathbb{R}^{q \times r}$ and $\mathbb{Z} \in \mathbb{S}^{r}$ that simultaneously satisfy the constraints (22)-(23) and (27)-(28), then the $\{\mathbb{X}, \mathbb{Y}, \mathbb{Z}\}$-ellipsoid is a set of stabilizing gains such that the performance levels are satisfied.

\section{Computational Issues and Example}

\subsection{A Cone Complementary (CCL) Algorithm}

The numerical example is solved using a first order iterative algorithm. It is based on a cone complementary technique [14], that allows to concentrate the non convex constraint in the criterion of some optimisation problem.

Lemma 1. The multi-objective control problem is feasible if and only if zero is the global optimum of the optimisation problem

$$
\left\{\begin{array}{l}
\min \operatorname{tr}(\mathcal{T} \mathcal{S}) \\
\text { s.t. } \quad(22),(27)-(28) \\
\mathbb{X} \leq \widehat{\mathbb{X}} \quad \mathcal{S}=\left[\begin{array}{cc}
\widehat{\mathbb{X}} & \mathbb{Y} \\
\star & \mathbb{Z}
\end{array}\right] \geq 0 \\
\mathcal{T}_{1} \geq \mathbb{I} \quad \mathcal{T}=\left[\begin{array}{ll}
\mathcal{T}_{1} & \mathcal{T}_{2} \\
\star & \mathcal{T}_{3}
\end{array}\right] \geq 0
\end{array}\right.
$$

Proof. The proof of this Lemma follows the same arguments as in [24].

CCL algorithm. For two given levels $\gamma_{\infty}>0, \gamma_{2}>0$

i) Find a feasible solution $\mathbb{X}_{0}, \mathbb{Y}_{0}, \mathbb{Z}_{0}, \hat{\mathbb{X}}_{0}, \mathcal{Q}_{i j 0}, \mathcal{T}_{0}, \mathcal{S}_{0}$. If there is no solution, STOP, the algorithm failed. $h=0$;

ii) set $\mathbf{V}_{h}=\mathcal{S}_{h}, \mathbf{W}_{h}=\mathcal{T}_{h}$, and find $\mathbb{X}_{(h+1)}, \mathbb{Y}_{(h+1)}, \mathbb{Z}_{(h+1)}, \hat{\mathbb{X}}_{(h+1)}, \mathcal{Q}_{i j(h+1)}, \mathcal{T}_{h+1}, \mathcal{S}_{(h+1)}, \mathbb{T}_{h+1}$ solutions of the LMI problem

$$
\begin{cases}\min & \operatorname{tr}\left(\mathbf{V}_{h} \mathcal{T}+\mathbf{W}_{h} \mathcal{S}\right) \\
\text { s.t. } & (22),(27)-(28) \\
\mathbb{X} \leq \widehat{\mathbb{X}} & \mathcal{S}=\left[\begin{array}{cc}
\widehat{\mathbb{X}} & \mathbb{Y} \\
\star & \mathbb{Z}
\end{array}\right] \geq 0 \\
\mathcal{T}_{1} \geq \mathbb{I} & \mathcal{T}=\left[\begin{array}{ll}
\mathcal{T}_{1} & \mathcal{T}_{2} \\
\star & \mathcal{T}_{3}
\end{array}\right] \geq 0\end{cases}
$$

iii) if $\operatorname{tr}\left(\mathcal{T}_{h-1} \mathcal{S}_{h-1}-\mathcal{T}_{h} \mathcal{S}_{h}<\varepsilon\right)$, then STOP, the algorithm failed.

iv) if $\mathbb{X} \leq \mathbb{Y} \mathbb{Z}^{-1} \mathbb{Y}^{\prime}$, STOP, a matrix ellipsoid is found. Otherwise, set $h=h+1$ and go to step ii). 


\subsection{Numerical Example}

In this section, the proposed static output feedback multi-objective control of the NCS subject to random failures is illustrated using a VTOL helicopter model [17]. The sampling time is $T_{s}=0.01 s$, and the random sensor delay exists in $r_{s} \in\{0,1\}$, and its transition probability matrix is given by

$$
\left[p_{i j}\right]=\left[\begin{array}{ll}
0.9 & 0.1 \\
0.9 & 0.1
\end{array}\right]
$$

Consider the nominal system with

$$
\begin{gathered}
A_{x}=\left[\begin{array}{cccc}
0.9996 & 2.70 \times 10^{-4} & 1.646 \times 10^{-4} & -4.557 \times 10^{-3} \\
4.794 \times 10^{-4} & 0.9900 & -1.761 \times 10^{-4} & -0.0400 \\
9.995 \times 10^{-4} & 0.0050 & 0.9931 & 0.0252 \\
0 & 0 & 9.965 \times 10^{-3} & 1
\end{array}\right], \\
B_{u}=\left[\begin{array}{cc}
4.423 \times 10^{-3} & 1.754 \times 10^{-3} \\
0.0508 & -0.0755 \\
-0.0548 & 0.0445 \\
-2.749 \times 10^{-4} & 2.233 \times 10^{-3}
\end{array}\right], E_{w}=\left[\begin{array}{cc}
0.0100 & 0 \\
0 & 0 \\
0 & 0 \\
0 & 3 \times 10^{-3}
\end{array}\right], C_{y}=\left[\begin{array}{llll}
0 & 0 & 0 & 1
\end{array}\right], \\
C_{\infty}=\left[\begin{array}{llll}
0 & 0 & 1 & 0 \\
0 & 0 & 0 & 0
\end{array}\right], D_{\infty}=\left[\begin{array}{ll}
0 & 0 \\
1 & 0
\end{array}\right], C_{2}=\left[\begin{array}{llll}
0 & 1 & 0 & 0 \\
0 & 0 & 0 & 0
\end{array}\right], D_{2}=\left[\begin{array}{ll}
0 & 0 \\
0 & 1
\end{array}\right] .
\end{gathered}
$$

For illustration purposes, we will consider the following faulty mode

○ Mode 2: Total loss of the second actuator.

From above, we have that $\beth=\{1,2\}$, where the mode 1 represents the nominal case. The failure process is assumed to have Markovian transition characteristics.

The actuator failure transition probability matrix is assumed to be:

$$
\left[\alpha_{i j}\right]=\left[\begin{array}{cc}
0.9 & 0.1 \\
0 & 1
\end{array}\right]
$$

Figure 2 shows the ellipsoidal set of controllers corresponding to $\gamma_{\infty}^{2}=\gamma_{2}^{2}=5$. The central controller (center of the ellipsoid) is given by

$$
\mathcal{K}_{0}^{\prime}=\left[\begin{array}{ll}
1.0575 & -0.1174
\end{array}\right]
$$

The state trajectories of the closed loop system resulting from the discretized model and the obtained controller are shown in figure 3. These trajectories represent a single sample path simulation corresponding to a realization of the failure process $\eta_{k}$ and the random delay process $r_{s k}$. Figure 4 represents the evolution of the controlled outputs $z_{k}$. It can be seen that the closed-loop system is stochastically stable and that the disturbance attenuation is achieved.

\section{Conclusion}

In this paper, the static output feedback multi-objective control of discrete-time NCSs subject to random failures and random delays was considered under the discrete-time Markovian Jump Linear Systems framework. The specifications and objectives considered include stochastic stability, $\mathcal{H}_{2}$ and $\mathcal{H}_{\infty}$ performances. The numerical resolution of the obtained results was done using a cone complementary algorithm and its running was illustrated on a numerical example. 


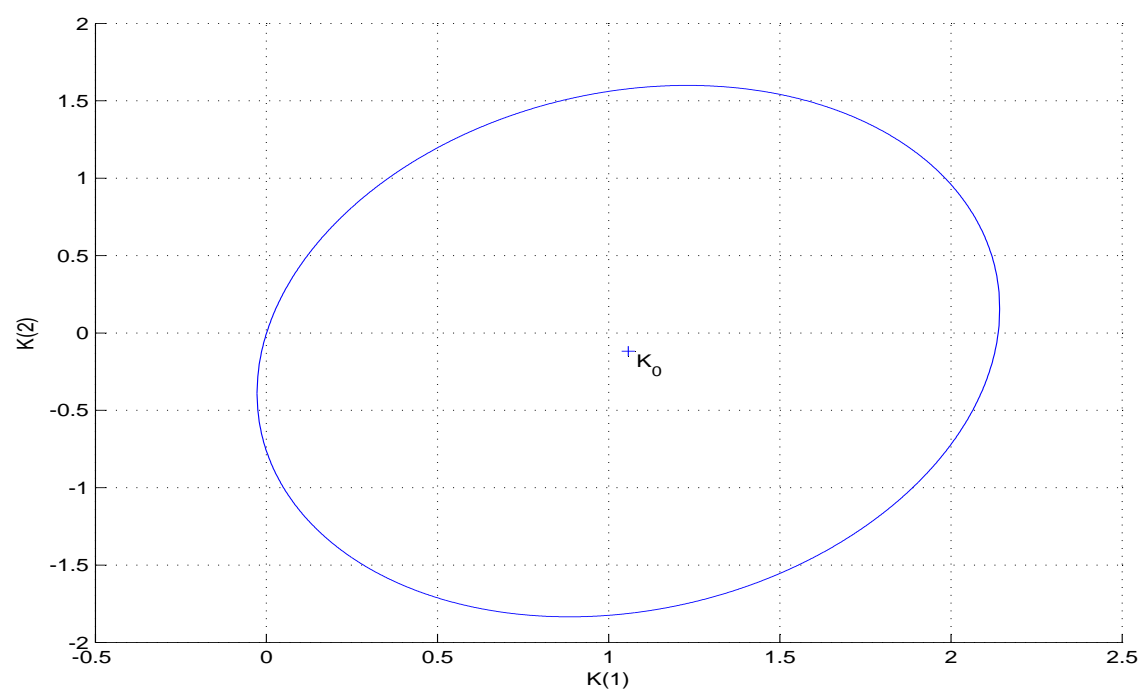

Figure 2: Static output feedback ellipsoid

\section{References}

[1] S. Aberkane, J.C. Ponsart and D. Sauter, Output Feedback Stochastic Stabilization of Active Fault Tolerant Control Systems: LMI Formulation, 16th IFAC World Congress, Prague, Czech Republic, 2005.

[2] S. Aberkane, D. Sauter and J.C. Ponsart, $\mathcal{H}_{\infty}$ Stochastic Stabilization of Active Fault Tolerant Control Systems: Convex Approach, 44th IEEE Conference on Decision and Control and European Control Conference ECC 2005, Seville, Spain, 2005.

[3] S. Aberkane, J.C. Ponsart and D. Sauter, Output Feedback $\mathcal{H}_{\infty}$ Control of a Class of Stochastic Hybrid Systems with Wiener Process via Convex Analysis, International Journal of Innovative Computing, Information and Control, Vol 2, No 6, 2006.

[4] E. K. Boukas, Static Output Feedback Control for Stochastic Hybrid Systems: LMI Approach, Automatica, Vol.42, pp.183-188, 2006.

[5] E. K. Boukas, Stabilization of Stochastic Nonlinear Hybrid Systems, Int. J. Innovative Computing, Information and Control, Vol.1, No.1, pp.131-141, 2005.

[6] E. K. Boukas, Exponential stabilizability of stochastic systems with Markovian jumping parameters, Automatica, Vol.35, pp.1437-1441, 1999.

[7] O. L. V. Costa and R. P. Marques, Mixed $H_{2} / H_{\infty}$ Control of Discrete-Time Markovian Jump Linear Systems, IEEE Transactions on Automatic Control, Vol 53, pp 95-100, 1998.

[8] O. L. V. Costa, J. B. R. do Val and J. C. Geromel, Continuous-time state-feedback $\mathcal{H}_{2}$-control of Markovian jump linear systems via convex analysis, Automatica, Vol 35, pp 259-268, 1999.

[9] O. L. V. Costa, M. D. Fragoso and R. P. Marques, Discrete-Time Markov Jump Linear Systems, Springer, 2005. 


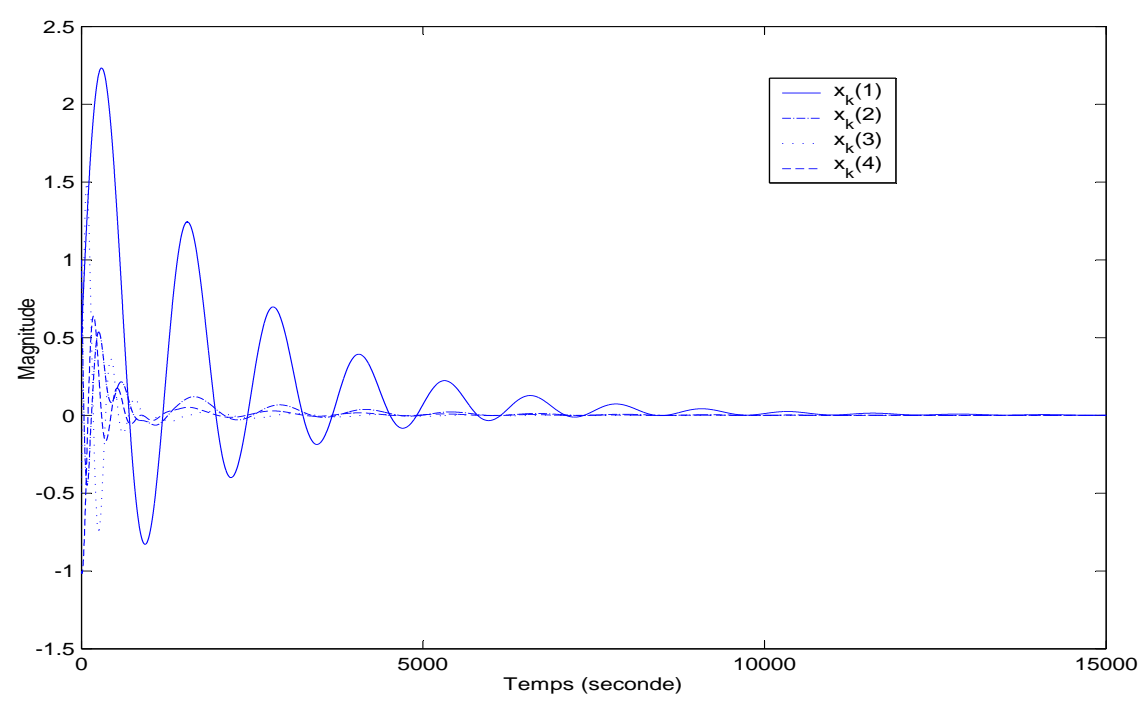

Figure 3: States of the closed loop system: single sample path simulation

[10] D. P. de Farias, J. C. Geromel, J. B. R. do Val and O. L. V. Costa, Output Feedback Control of Markov Jump Linear Systems in Continuous-Time, IEEE Transactions on Automatic Control, Vol 45, pp 944-949, 2000.

[11] C. E. de Souza and M. D. Fragoso, $\mathcal{H}_{\infty}$ Control For Linear Systems With Markovian Jumping Parameters, Control Theory and Advanced Technology, Vol 9, No. 2, pp 457-466, 1993.

[12] V. Dragan and T. Morozan, Stability and Robust Stabilization to Linear Stochastic Systems Described by Differential Equations with Markov Jumping and Multiplicative White Noise, Stochastic Analysis and Applications, Vol 20, pp 33-92, 2002.

[13] V. Dragan, T. Morozan and A. Stoica, $\mathcal{H}_{2}$ Optimal Control for Linear Stochastic Systems, Automatica, Vol 40, pp 1103-1113, 2004.

[14] L. El Ghaoui, F. Oustry and M. AitRami, A Cone Complementary Linearization Algorithm for Static Output-Feedback and Related Problems, IEEE Transactions on Automatic Control, Vol 42, No. 8, pp 1171-1176, 1997.

[15] Y. Ji and H. J. Chizeck, Controllability, stabilizability, and continuous-time Markovian jump linear quadratic control, IEEE Transactions on Automatic Control, Vol 35, pp 777-788, 1990.

[16] Y. Ji and H. J. Chizeck, Jump linear quadratic Gaussian control in continuous time, IEEE Transactions on Automatic Control, Vol 37, pp 1884-1892, 1992.

[17] B. Jiang and F. N. Chowdhury, Fault Estimation and Accomodation for Linear MIMO DiscreteTime Systems, IEEE Transactions on Control Systems Technology, Vol 13, pp 493-499, 2005.

[18] L. H. Keel and S. P. Bhattacharyya, Robust, Fragile or Optimal, IEEE Transactions on Automatic Control, Vol 42, pp 1098-1105, 1997. 


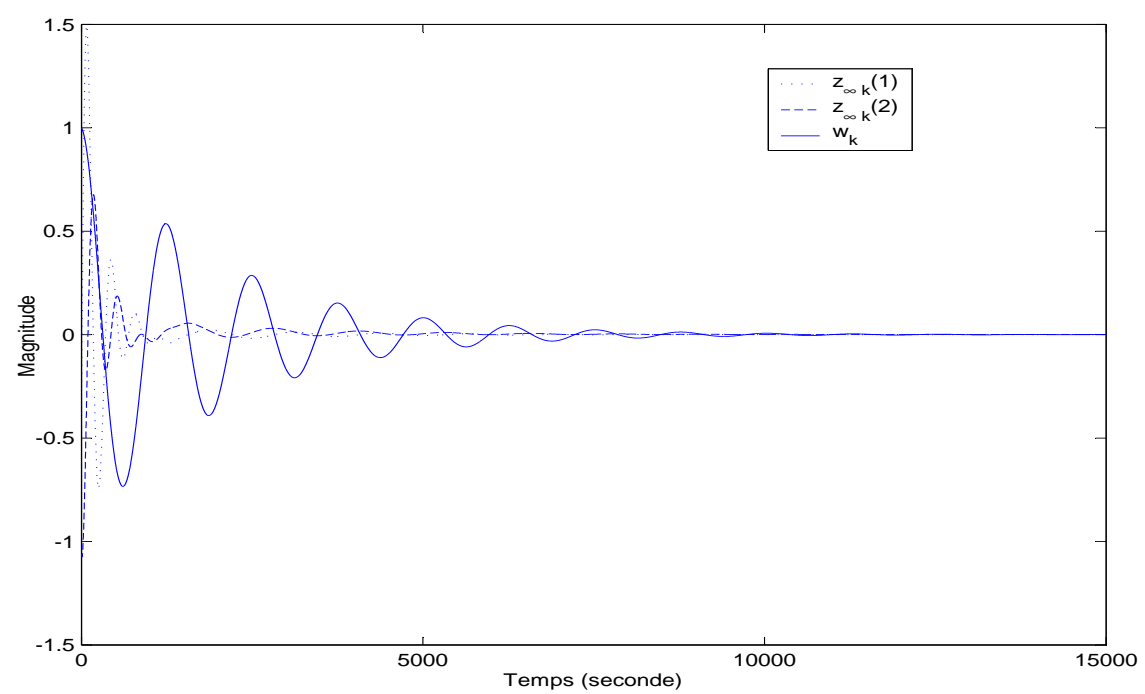

Figure 4: Evolution of the controlled output $z_{\infty k}$ : single sample path simulation

[19] R. Krtolica, U. Ozguner, H. Chan, H. Goktas, J. Winkelman and M. Liubakka, Stability of Linear Feedback Systems with Random Communication Delays, International Journal of Control, Vol 37, pp 1884-1892, 1992.

[20] H. Lin, G. Zhai and P. Antsaklis, Robust Stability and Disturbance Attenuation Analysis of a Class of Networked Control Systems, Proceedings of the $42^{\text {nd }}$ IEEE Conference on Decision 8 Control, Maui, HI, pp 1182-1187, 2003.

[21] M. Mahmoud, J. Jiang and Y. Zhang, Active Fault Tolerant Control Systems: Stochastic Analysis and Synthesis, Springer, 2003.

[22] J. Nilsson, B. Bernhardson and B. Wittenmark, Stochastic Analysis and Control of Real-Time Systems with Random Time Delays, Automatica, Vol 34, No. 1, pp 57-64, 1998.

[23] D. Peaucelle, D. Arzelier and R. Bertrand, Ellipsoidal Sets for Static Output-Feedback, 15th IFAC World Congress, Barcelona, Spain, 2002.

[24] D. Peaucelle and D. Arzelier, Ellipsoidal Sets for Resilient and Robust Static Output-Feedback, IEEE Transaction on Automatic Control, Vol 50, No. 6, pp 899-904, 2005.

[25] P. Shi and E. K. Boukas, $\mathcal{H}_{\infty}$-Control for Markovian Jumping Linear Systems with Parametric Uncertainty, Journal of Optimization Theory and Applications, Vol 95, pp 75-99, 1997.

[26] P. Shi, E. K. Boukas, S. K. Nguang and X. Guo, Robust disturbance attenuation for discretetime active fault tolerant control systems with uncertainties, Optimal Control Applications and Methods, Vol 24, pp 85-101, 2003.

[27] R. E. Skelton, T. Iwasaki and K. Grigoriadis, A Unified Algebraic Approach to Linear Control Design, Taylor and Francis, 1998.

[28] R. Srichander and B. K. Walker, Stochastic stability analysis for continuous-time fault tolerant control systems, International Journal of Control, Vol 57, pp 433-452, 1993. 
[29] V. L. Syrmos, C. T. Abdallah, P. Dorato and K. Grigoriadis, Static Output Feedback: A Survey, Automatica, Vol 33, No. 2, pp 125-137, 1997.

[30] C. C. Walsh, H. Ye and L. G. Bushnell, Stability Analysis of Networked Control Systems, IEEE Transaction on Control Systems Technology, Vol 10, No. 3, 2002, pp 438-446.

[31] L. Xiao, A. Hassibi and J. P. How, Control with Random Communication Delays via Discretetime Jump Linear System Approach, Proceedings of the 2000 American Control Conference, pp 2199-2204, 2000.

[32] G. Xie and L. Wang, Stabilization of Networked Control Systems with Time-Varying NetworkInduced Delay, Proceedings of the $43^{\text {rd }}$ IEEE Conference on Decision 86 Control, pp 3551-3556, 2004.

[33] M. Yu, L. Wang, T. Chu ang G. Xie, Stabilization of Networked Control Systems with Data Packet Dropout and Network Delays via Switching System Approach, Proceedings of the $43^{\text {rd }}$ IEEE Conference on Decision \& Control, pp 3539-3544, 2004.

[34] L. Zhang, B. Huang and J. Lam, $\mathcal{H}_{\infty}$ Model Reduction of Markovian Jump Linear Systems, Systeme and Control Letters, Vol 50, pp 103-118, 2003.

[35] L. Zhang, Y. Shi, T. Chen and B. Huang, A New Method for Stabilization of Networked Control Systems with Random Delays, IEEE Transactions on Automatic Control, Vol 50, No. 8, pp 1177-1181, 2005.

[36] W. Zhang, M. S. Branicky and S. M. Phillips, Stability of Networked Control Systems, IEEE Control System Magazine, Vol 21, No. 2, 2001, pp 84-99.

[37] Y. Zhang and J. Jiang, Bibliographical review on reconfigurable fault-tolerant control systems, IFAC SAFEPROCESS, pp 265-276, 2003. 